\title{
Plasma-Grafting of Polymers with Pendant Oligo(oxyethylene) Chains onto the Surface of a Polyaniline Electrode
}

\author{
Kenji Yamada, Akiko Ito, Naoya Iwamoto, \\ Toshihide HARAgUCHI, and Tisato KaJIYAMA* \\ Department of Materials Science and Chemical Engineering, \\ Kitakyusyu National College of Technology, Kitakyusyu 802-0985, Japan \\ *Department of Materials Physics and Chemistry, Graduate School of Engineering, \\ Kyushu University, Fukuoka 812-8581, Japan
}

(Received July 12, 1999)

\begin{abstract}
This paper reports experiments on forming a layer composed of graft polymers with pendant oligo(oxyethylene) chains directly onto the surface of a polyaniline film by plasma-graft polymerization. The graft polymers were covalently bonded with polyaniline film. Vinyl monomer with pendant oligo(oxyethylene) chain was synthesized and used for plasma-graft polymerization. The polyaniline film was irradiated with argon plasma to form active radicals on its surface, and then immersed in monomer solution to graft polymerize at $293 \mathrm{~K}$. A graft layer composed of linear polymers with pendant oligo(oxyethylene) chains was formed on the surface of the film and the average thickness of the graft layer increased with polymerization time and concentration of monomer solution. A graft layer of crosslinked polymers was also formed using the vinyl monomer and divinyl monomer having an oligo(oxyethylene) chain. The graft layer composed of crosslinked polymers became ion-conductive by inclusion of $\mathrm{LiClO}_{4}$. The interface resistance between the polyaniline film and graft layer decreased, compared with that between the film and freestanding polymer solid electrolyte photopolymerized with the same compositions of monomers as the graft layer.

KEY WORDS Plasma-Graft Polymerization / Polyaniline / Surface / Polymer Solid Electrolyte / Oligo(oxyethylene) Chain / Interface Resistance / Ionic Conductivity /
\end{abstract}

Plasma-graft polymerization is effective for modifying the surface of polymeric materials. Yamada et al. ${ }^{1}$ previously reported experiments in which the surface of poly( $p$-phenylene terephthalamide) fiber was modified to obtain strong adhesion between the fiber and matrix resin by plasma-graft polymerization. The vinyl monomer had a functional group which reacted with the matrix resin to form covalent bonds between the plasma-grafted fiber and matrix resin. ${ }^{1}$ Poly(vinylsulfonic acid) chains having cation-exchangeable groups formed onto the surface of polyaniline film by plasma-graft polymerization, ${ }^{2,3}$ and the plasma-grafting was effective for inducing cation migration which takes place to maintain electroneutrality of the polyaniline film during redox in an electrolyte. ${ }^{2,3}$

Comblike polymers containing oligo(oxyethylene) chains and those copolymerized with alkali-metal methacrylates have been used as matrix polymers of polymer solid electrolytes. ${ }^{4-13}$ The latter copolymers have prepared polymeric solid electrolytes with single-ion conductor properties. Polymer electrolytes based on a networkpolymer of poly(glycidylether) have been also investigated. ${ }^{14}$ When a polymeric solid electrolyte is used to a secondary battery composed of a conducting polymer and $\mathrm{Li}$ as a cathode and an anode, respectively, good interfacial contact of the conducting polymer/the polymer electrolyte and optimum thinning of the polymer electrolyte are necessary to lowering inner resistance and reproducible redox behavior. If covalent bonds are formed between the electrolyte and polymer electrode, the interface adheres more closely and therefore the interface resistance becomes lower. This work therefore investigated how to form comb polymers with pendant oligo(oxyethylene) chains onto the surface of polyaniline film by plasma-graft polymerization. Vinyl monomer with pendant oligo(oxyethylene) chain is synthesized for use in plasma-graft polymerization and the effects of plasma-graft polymerization conditions on the degree of grafting are described. Ionic conductivity of the graft layer composed of the comb polymer crosslinked by oligo(oxyethylene) chain is clarified. The interface resistance between the polyaniline film and graft layer is evaluated to clarify the effects of graft polymerization on lowering the resistance.

\section{EXPERIMENTAL}

\section{Synthesis of Vinyl Monomer}

According to Figure 1, vinyl monomer was synthesized by reacting glycidyl methacrylate (hereafter abbreviated GMA) with poly(ethylene glycol) methyl ether (hereafter abbreviated PEGME). The molecular weight of PEGME was 350 and average number of oxyethylene unit, 7.2. The number of moles of PEGME in the synthesis was the same as that of GMA. GMA was reacted at $363 \mathrm{~K}$ for $7 \mathrm{~h}$ in an atmosphere of nitrogen with PEGME using po-

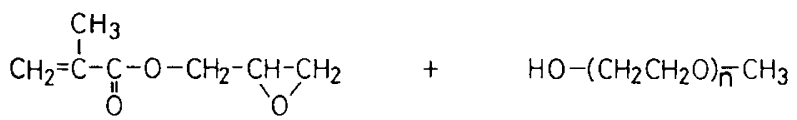

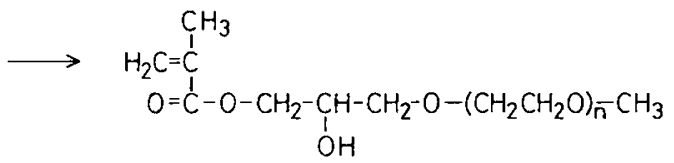

Figure 1. Synthetic scheme of vinyl monomer with pendant oligo(oxyethylene) chain. 


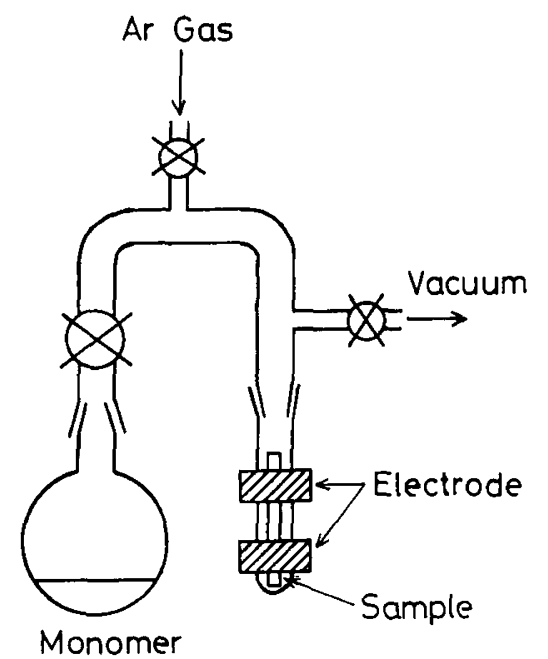

Figure 2. Schematic representation of apparatus for plasmagraft polymerization.

tassium hydroxide as a catalyst. Potassium hydroxide was $4 \mathrm{~mol} \%$ to GMA. The synthesized monomer was extracted with benzene to remove potassium hydroxide and recovered by evaporating benzene. The synthesized monomer was identified by a Nihondenshi FX 90Q nuclear magnetic resonance spectroscopy (Nihondenshi Co., Ltd.).

\section{Plasma-Graft Polymerization}

Polyaniline film was electropolymerized by galvanostatic electrolysis at current density $5 \mathrm{~mA} \mathrm{~cm}^{-2}$ in the electrolyte which was $2.37 \mathrm{M} \mathrm{HClO}_{4}$ aqueous solution containing $0.52 \mathrm{M}$ aniline. The electrode assembly consisted of a stainless steel plate as a working electrode, $\mathrm{Ag} / \mathrm{AgCl}$ electrode as a reference electrode, and a platinum plate as a counter electrode. The charge consumed during electropolymerization was $2 \mathrm{C} \mathrm{cm}^{-2}$. The electropolymerized film was washed successively with pure water and methanol, and dried in vacuum at $293 \mathrm{~K}$ for 1 day. Figure 2 shows a schematic representation of the apparatus for plasma-graft polymerization. The synthesized monomer was dissolved by methanol/water $(95 / 5)$ mixture. The monomer solution was freeze-vaporized to remove dissolved oxygen gas before use in plasma-graft polymerization. The concentrations of monomer solution were 0.73 to $1.32 \mathrm{M}$. The film was irradiated for $60 \mathrm{~s}$ with plasma generated at $13.56 \mathrm{MHz}$ at $50 \mathrm{~W}$ in an atmosphere of $13 \mathrm{~Pa}$ argon, and the monomer solution was poured into the sample vessel. The film was immersed in monomer solution to graft polymerize at $293 \mathrm{~K}$ over periods of $4 \mathrm{~h}$ to $48 \mathrm{~h}$. The film was washed with methanol after plasma-grafting and then was dried in vacuum at $293 \mathrm{~K}$ for 1 day. In a similar manner, polyaniline film was plasma-graft polymerized at $293 \mathrm{~K}$ for $48 \mathrm{~h}$ using $1.32 \mathrm{M}$ vinyl monomer solution containing $1 \mathrm{~mol} \%$ poly(ethylene glycol)dimethacrylate with number-average molecular weight of 536, to form crosslinked graft polymers. Inhibitors in poly(ethylene glycol)dimethacrylate supplied from Aldrich Chemical Co., Inc. were removed by inhibitor remover (Aldrich Chemical Co., Inc.) before use in the plasma-graft polymerization.

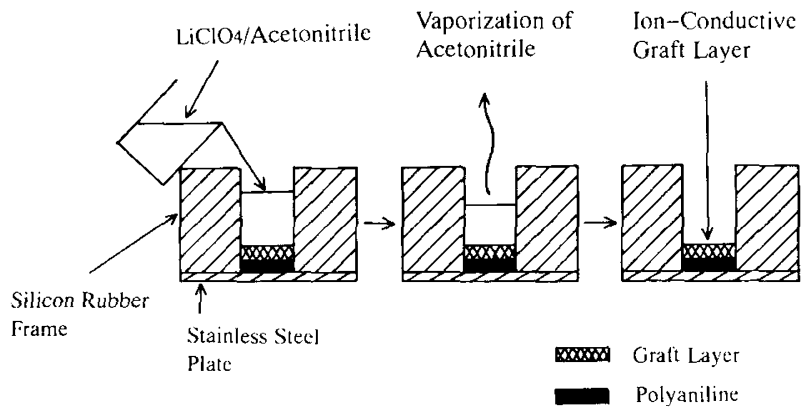

Figure 3. Schematic representation of preparation method of ionconductive graft layer on the surface of polyaniline film.

\section{Surface Analyses of Plasma-Grafted Film}

$\mathrm{X}$-ray photoelectron spectroscopy (XPS) measurement was carried out with a Shimadzu ESCA750 X-ray photoelectron spectroscopy (Shimadzu Co., Ltd.) to clarify the species of atoms constituting the graft layer. An infrared spectrum was measured with a Nicolet Magna 550 Fourier transform infrared spectroscopy (Nicolet Analytical Instruments) equipped with a Barnes analytical diffuse reflectance accessory to clarify chemical structure of the graft layer. The surface morphology of the plasma-grafted film was observed by a Nihondenshi JSM-6301 FZ scanning electron microscope (Nihondenshi Co., Ltd.).

Evaluation of Ionic Conductivity and Interface Resistance

Figure 3 shows a schematic representation of the method for introducing $\mathrm{LiClO}_{4}$ into crosslinked graft layer formed on a surface of polyaniline film. Acetonitrile solution $0.33 \mathrm{M}$ of $\mathrm{LiClO}_{4}$ was poured on the grafted polyaniline film surrounded by a frame of silicon rubber and then vaporized at $293 \mathrm{~K}$ to form the solid electrolyte composed of grafted chains and $\mathrm{LiClO}_{4}$ on the polyaniline film. The mole ratio of $\mathrm{LiClO}_{4}$ to oxyethylene units in pendant oligo(oxyethylene) chains and crosslinking chains, $\mathrm{LiClO}_{4} /\left(\mathrm{CH}_{2} \mathrm{CH}_{2} \mathrm{O}\right)$ was controlled by the amount of solution of $\mathrm{LiClO}_{4}$ depending on the weight of graft layer and was 0.03 to 0.15 in the electrolyte layer. Infrared spectra of upper and lower surfaces of the graft layer from which the polyaniline film was removed were measured by diffuse reflectance to investigate the distribution of $\mathrm{LiClO}_{4}$ in the layer. Absorbance $\mathrm{A}_{1}$ of $\mathrm{Cl}-\mathrm{O}$ stretching vibration of $\mathrm{ClO}_{4}{ }^{-}$ions was evaluated with that $A_{2}$ of $C=O$ stretching vibration of graft chains. $A_{1} /$ $\mathrm{A}_{2}$ on the lower surface was almost same as that on the upper surface. Therefore it was assumed that $\mathrm{LiClO}_{4}$ was almost uniformly distributed in the graft layer. The polyaniline films having the electrolyte layer were combined to evaluate ionic conductivity of the layer, in such a way that the graft layers were in contact with each other. The combined films were dried in vacuum at $293 \mathrm{~K}$ for 1 day before measurement of complex impedance. The complex impedances of the combined films were measured at $293 \mathrm{~K}$ in the frequency range of $1 \mathrm{mHz}$ to $5 \mathrm{MHz}$ with a Hioki LCR Hi Tester 3522 and 3531 impedance measurements (Hioki Denki Co., Ltd.). The resistance of the graft layers and interface resistance between the film and graft layer were evaluated from impedance plots. The ionic conductivity $\sigma$ of the graft layers was cal- 


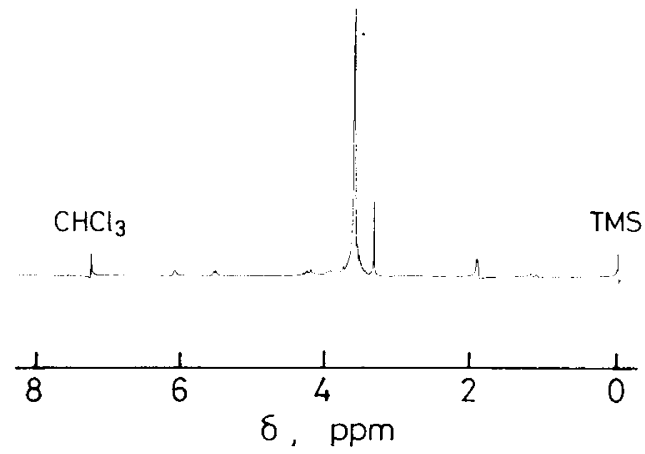

Figure 4. NMR spectrum of the synthetic vinyl monomer.

culated from eq 1 :

$$
\sigma=d / R_{\mathrm{b}} A
$$

where $R_{\mathrm{b}}$ is the resistance of the graft layers, $d$ thickness of the graft layers, and $A$ area of the graft layers. The thickness $d$ of the graft layers was evaluated by subtracting the thickness of stainless steel electrodes and polyaniline films from total thickness of sample assembly composed of graft layers sandwiched with the polyaniline films and stainless steel electrodes. Thickness was measured with a micrometer.

Interface resistance between the polyaniline film and freestanding polyelectrolyte film was also evaluated from impedance plots to compare with that between the polyaniline film and graft layer. The freestanding film was prepared as follows: Synthesized monomer containing $1 \mathrm{~mol} \%$ poly(ethylene glycol)dimethacrylate poured in a frame located on a stainless steel plate was irradiated for $2 \mathrm{~h}$ with a mercury lamp of $100 \mathrm{~W}$ and then acetonitrile solution $0.33 \mathrm{M}$ of $\mathrm{LiClO}_{4}$ was poured upon the mixture. The mixture was polymerized at 293 $\mathrm{K}$ for $48 \mathrm{~h}$ to form a film and dried in vacuum at $293 \mathrm{~K}$ for 1 day.

\section{RESULTS AND DISCUSSION}

\section{Identification of Synthetic Vinyl Monomer}

Figure 4 shows ${ }^{1} \mathrm{H}$ NMR spectrum of the vinyl monomer synthesized by GMA and PEGME. The peaks at 2.8 ppm and $3.3 \mathrm{ppm}$ are associated with epoxy group and disappeared in the monomer. The peaks at $3.4 \mathrm{ppm}$ and $3.7 \mathrm{ppm}$ are associated with methyl and methylene groups of PEGME, and those at $5.6 \mathrm{ppm}$ and $6.2 \mathrm{ppm}$, the vinyl group. The proportion of peak area of $3.4 \mathrm{ppm}$ to $3.7 \mathrm{ppm}$ was almost the same as that of PEGME. The epoxy group of GMA thus almost reacts with hydroxy group of PEGME and is hardly ring-opened polymerized. Since a small peak at $1.2 \mathrm{ppm}$ is associated with methylene group of the main chain formed by polymerization, vinyl polymerization slightly takes place at the same time. Based on peak areas of $1.2 \mathrm{ppm}, 5.6 \mathrm{ppm}$, and 6.2 ppm per cent polymerization was $15 \%$.

\section{Plasma-Graft Polymerization and Ionic Conductivity of Graft Layer}

Figure 5 shows scanning electron micrograph (SEM) photographs of polyaniline film before and after plasmagraft polymerization. Microfibrillar aggregation was ob-

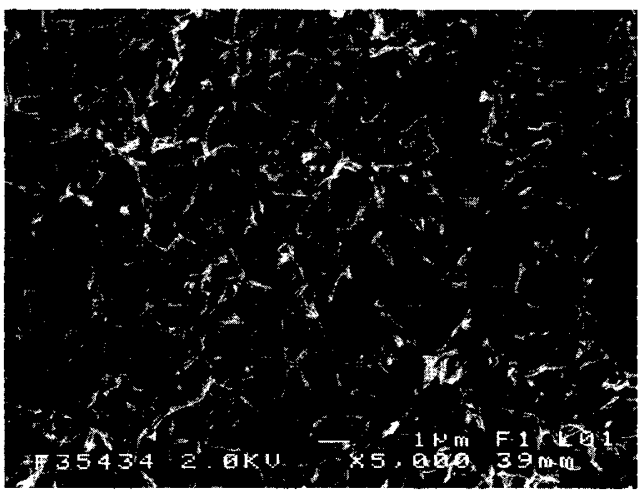

(a)

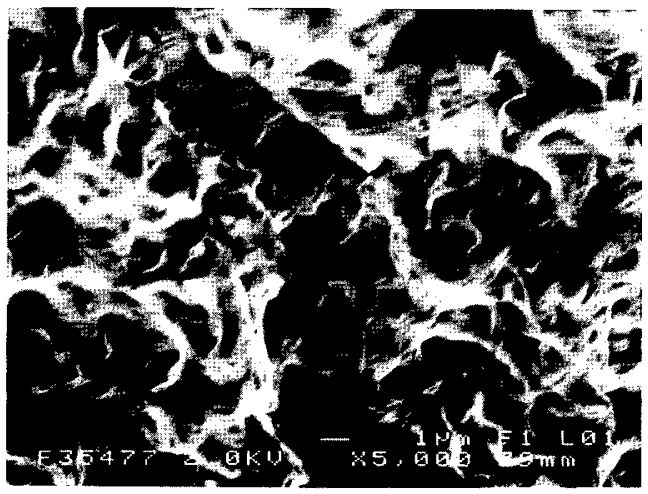

(b)

Figure 5. Scanning electron micrographs of polyaniline film (a) before and (b) after plasma-graft polymerization (concentration of monomer solution was $1.32 \mathrm{M}$ and polymerization time was $43 \mathrm{~h}$ ).

served before plasma-grafting. However after plasmagrafting the surface morphology was largely changed and microfibrillar aggregation was not found. Figure 6 shows the XPS spectra of polyaniline film before and after plasma-graft polymerization. Peaks of $\mathrm{C}_{1 \mathrm{~s}}, \mathrm{~N}_{1 \mathrm{~s}}, \mathrm{Cl}_{2 \mathrm{p}}$, and $\mathrm{O}_{1 \mathrm{~s}}$ were recognized before plasma-grafting. The peaks of $\mathrm{C}_{1 \mathrm{~s}}$ and $\mathrm{N}_{1 \mathrm{~s}}$ originated from polyaniline and the peaks of $\mathrm{Cl}_{2 \mathrm{p}}$ and $\mathrm{O}_{1 \mathrm{~s}}$ from $\mathrm{ClO}_{4}{ }^{-}$ions doped in the film. $\mathrm{N}_{1 \mathrm{~s}}$ and $\mathrm{Cl}_{2 \mathrm{p}}$ peaks disappeared after plasma-grafting. Figure 7 shows infrared spectrum on the surface of polyaniline film after plasma-graft polymerization. The absorption peaks associated with $\mathrm{C}-\mathrm{O}-\mathrm{C}$ symmetric stretching and $\mathrm{C}=\mathrm{O}$ stretching vibrations of ether and ester groups appeared at $1060 \mathrm{~cm}^{-1}$ and $1740 \mathrm{~cm}^{-1}$, respectively. Absorption peaks associated with $\mathrm{CH}$ out-of plane bending and $\mathrm{CH}$ stretching vibration of the vinyl group were not recognized. From Figures 5, 6, and 7, the polymers with pendant oligo(oxyethylene) chains are formed onto the surface of film by plasma-grafting of the synthesized vinyl monomer. This means that active radicals are formed on the surface of film by irradiation of argon plasma and vinyl monomers react with radicals to form graft chains.

Figure 8a shows XPS intensity ratio $\mathrm{N}_{1 \mathrm{~s}} / \mathrm{C}_{1 \mathrm{~s}}$ as a function of polymerization time, using $1.32 \mathrm{M}$ monomer solution. The intensity ratio $\mathrm{N}_{1 \mathrm{~s}} / \mathrm{C}_{1 \mathrm{~s}}$ corresponds to an atomic ratio $\mathrm{N} / \mathrm{C}$ in surface area of the film. $\mathrm{N}_{1 \mathrm{~s}} / \mathrm{C}_{1 \mathrm{~s}}$ decreased with increase in polymerization time. Figure $8 \mathrm{~b}$ shows $\mathrm{N}_{1 \mathrm{~s}} / \mathrm{C}_{1 \mathrm{~s}}$ as a function of the concentration of monomer so- 


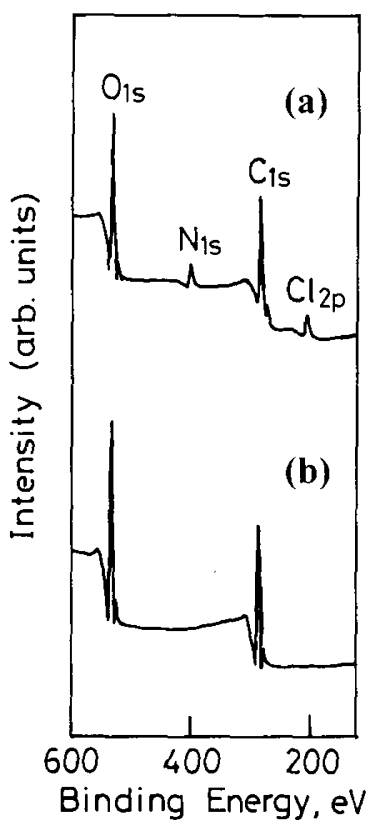

Figure 6. X-ray photoelectron spectra of polyaniline film (a) before and (b) after plasma-graft polymerization (concentration of monomer solution was $1.32 \mathrm{M}$ and polymerization time, $43 \mathrm{~h}$ ).

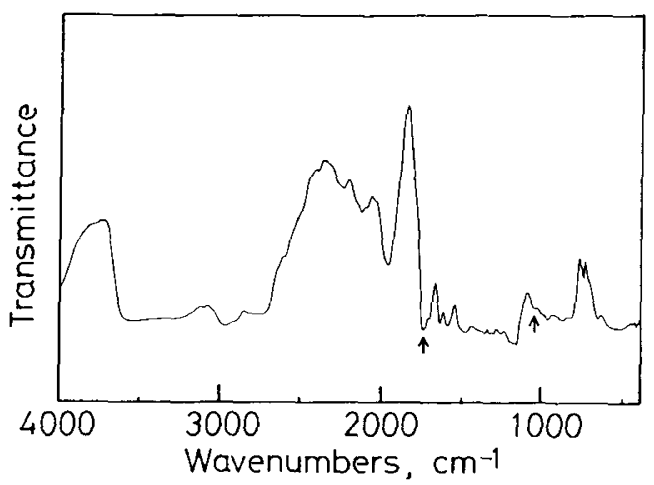

Figure 7. Infrared spectrum on the surface of polyaniline film after plasma-graft polymerization (concentration of monomer solution was $1.32 \mathrm{M}$ and polymerization time was $43 \mathrm{~h}$ ).

lution at polymerization time of $43 \mathrm{~h} . \mathrm{N}_{1 \mathrm{~s}} / \mathrm{C}_{1 \mathrm{~s}}$ decreased with increase in concentration, especially at a concentration of $1 \mathrm{M}$. This shows that the graft layer observed with a scanning electron microscope would be heterogeneously formed on a surface of the film ${ }^{3}$ and the fraction of the graft area below the concentration of the monomer of $0.93 \mathrm{M}$ would be less than $100 \%$. Above $1.1 \mathrm{M}$ the graft layer with thickness above that corresponding to the analytical depth of X-ray photoelectron spectroscopy completely covers the surface of the film by extending of the graft area. The results of Figures $8 \mathrm{a}$ and $8 \mathrm{~b}$ mean that the average thickness of the graft layer increases with polymerization time and concentration of the monomer solution and therefore average thickness of graft layer can be controlled by changing the polymerization time or/and the concentration.

From infrared spectrum of the surface of the grafted film, the crosslinked graft layer plasma-graft polymerized using the vinyl monomer solution containing $1 \mathrm{~mol}$ $\%$ poly(ethylene glycol)dimethacrylate sufficiently covers

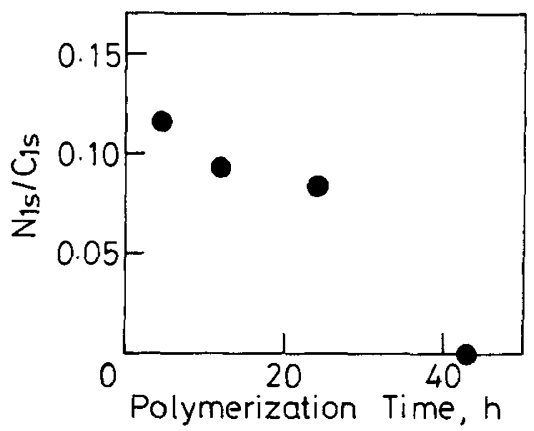

(a)

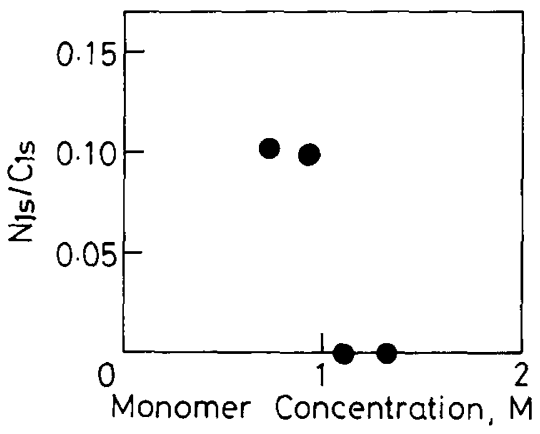

(b)

Figure 8. XPS intensity ratio $N_{1 s} / C_{1 s}$ as functions of (a) polymerization time at concentration of monomer solution, $1.32 \mathrm{M}$ and (b) concentration of monomer solution at polymerization time, $43 \mathrm{~h}$.

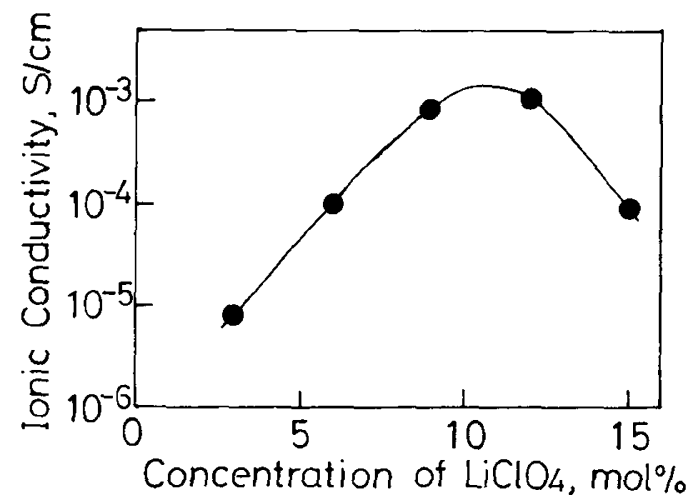

Figure 9. Ionic conductivity as a function of $\mathrm{LiClO}_{4}$ content for the graft layer composed of the comb polymer crosslinked by poly(ethylene glycol) chains (concentration of monomer solution was $1.32 \mathrm{M}$ and polymerization time, $48 \mathrm{~h}$ ).

the surface of the film. Ionic conductivity of the crosslinked graft layer was measured, since the uncrosslinked graft layer composed of only comb polymers became liquid-like by introducing $\mathrm{LiClO}_{4}$ and it was difficult to use as polymer solid electrolyte. Figure 9 shows ionic conductivity of the crosslinked graft layer as a function of $\mathrm{LiClO}_{4}$ content. Ionic conductivity was above $10^{-6} \mathrm{~S} \mathrm{~cm}^{-1}$ and therefore confirmed that the crosslinked graft layer becomes ionic conductive by containing $\mathrm{LiClO}_{4}$. Very high ionic conductivity of about $10^{-3} \mathrm{~S} \mathrm{~cm}^{-1}$ was obtained around $11 \mathrm{~mol} \% \mathrm{LiClO}_{4}$. It 


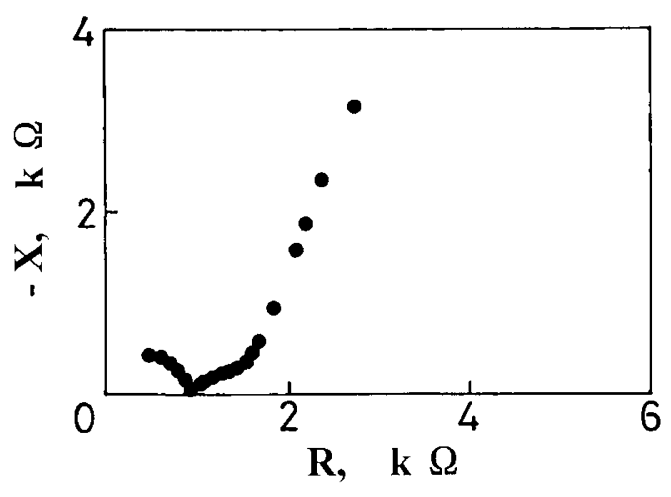

Figure 10. Impedance plots of the grafted polyaniline films combined in such a way that graft layers were in contact with each other (thickness of graft layers, $0.85 \mathrm{~mm}$ ).

was assumed that a slight amount of water remains in the graft layer even if the plasma-grafted polyaniline film is dried in vacuum at $293 \mathrm{~K}$ for 1 day after being washed with methanol, since the plasma-graft polymerization was carried out in methanol/water (95/5) mixture. This was confirmed by contact of the surface of the layer with a lithium sheet, since brightness of the lithium sheet was missed after the contact. Since ionic conductivity depends on concentration of ions and mobility of ions, it would be assumed that decrease of mobility of ions due to increase of ionic interactions brings about decrease of the ionic conductivity above $11 \mathrm{~mol} \%$.

\section{Interface Resistance between Polyaniline Film and Graft Layer}

Figure 10 shows impedance plots of the grafted polyaniline films combined in such a way that the graft layers are in contact with each other. The graft layer contained $15 \mathrm{~mol}_{\%} \mathrm{LiClO}_{4}$ to oxyethylene units in the pendant chains and the crosslinking chains. The area of the films was $1 \mathrm{~cm}^{2}$. The concentration of ions at $15 \mathrm{~mol} \%$ $\mathrm{LiClO}_{4}$ is the highest in the concentrations of 3 to $15 \mathrm{~mol}$ $\% \mathrm{LiClO}_{4}$. Therefore the concentration of $15 \mathrm{~mol} \% \mathrm{Li}$ $\mathrm{ClO}_{4}$ was adopted in the impedance measurement for evaluating interface resistance between polyaniline film and graft layer, since the interface resistance depends on how to move ions located around the interface. Resistance of the graft layer was $0.95 \mathrm{k} \Omega$ and interface resistance between polyaniline film and graft layer was 0.68 $\mathrm{k} \Omega$. The electric conductivity of polyaniline film of about $10 \mathrm{~S} \mathrm{~cm}^{-1}$ was much higher than ionic conductivity of the graft layer. The decrease in electric conductivity of the polyaniline film caused by the argon plasma irradiation was less than $10 \%$. Therefore the polyaniline film is electric conductive and behaves similar to non-blocking electrode in which $\mathrm{ClO}_{4}{ }^{-}$ions are doped into the polyaniline film or undoped from the film by alternating electric field. Figure 11 shows impedance plots of the freestanding crosslinked polymer film interposed between the polyaniline films before plasma-grafting. The freestanding film contained $15 \mathrm{~mol} \% \mathrm{LiClO}_{4}$. Resistance of the freestanding film was $11.1 \mathrm{k} \Omega$ and interface resistance between the polyaniline film and the freestanding film was $4.0 \mathrm{k} \Omega$. Interface resistance between the polyaniline film and graft layer was lower than that between the polyaniline film and the freestanding film. As shown

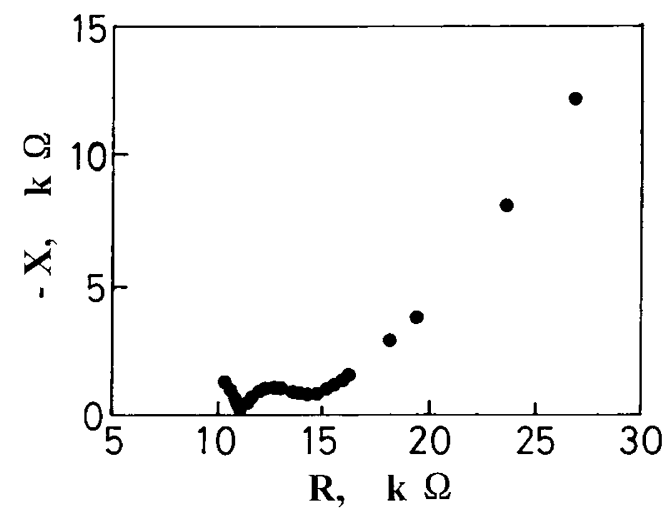

Figure 11. Impedance plots of the freestanding photopolymerized film interposed between polyaniline films before plasmagrafting (thickness of freestanding film, $0.40 \mathrm{~mm}$ ).

in Figure 5a, the surface of polyaniline film is rough, because of the formation of microfibrillar aggregation. Therefore contact between the free-standing film and polyaniline film is restricted in measurement of impedance and a net contact area decreases. Since graft polymers are formed on the surface regions irradiated by argon plasma, the graft layer is formed, so as to fill up the holes on a surface of polyaniline film, and adheres more closely with the polyaniline film by the formation of covalent bonds on the interface. According to eq 1, the conductivity of the graft layers was 25 times that of the bulk polymer. Difference in resistance between the graft layer and bulk polymer would be due to differences in concentration of unremoved water and molecular aggregation state which may depend on polymerization method.

\section{CONCLUSION}

The graft layer composed of polymers with pendant oligo(oxyethylene) chains can be formed on polyaniline film by plasma-graft polymerization. The average thickness of the graft layer increases with polymerization time and concentration of the vinyl monomer solution. The graft layer composed of comb polymers crosslinked by poly(ethylene glycol) chains becomes ionic conductivity by containing $\mathrm{LiClO}_{4}$. Interface resistance between the polyaniline film and graft layer is lowered by increase in contact area and the formation of covalent bonds on the interface.

\section{REFERENCES}

1. K. Yamada, T. Haraguchi, and T. Kajiyama, J. Appl. Polym. Sci., 60, 1847 (1996).

2. K. Yamada, T. Haraguchi, and T. Kajiyama, Rept. Progr. Polym. Phys. Jpn, 38, 461 (1995).

3. K. Yamada, T. Haraguchi, and T. Kajiyama, Polym. J., 30, 133 (1998).

4. D. W. Xia, D. Soltz, and J. Smid, Solid State Ionics, 14, 221 (1984).

5. D. Fish, D. W. Xia, and J. Smid, J. Makromol. Chem., Rapid Commun., 6, 761 (1985).

6. D. J. Bannister, G. R. Davies, I. M. Ward, and J. F. McIntyre, Polymer, 25, 1600 (1984).

7. N. Kobayashi, T. Hamada, H. Ohno, and E. Tsuchida, Polym. $J ., 18,661$ (1986).

8. P. M. Blonsky, D. F. Shriver, P. Austin, and H. R. Allcock, J. 
Am. Chem. Soc., 106, 6854 (1984).

9. D. Fish, I. M. Khan, and J. Smid, J. Makromol. Chem., RapidCommun., 7, 115 (1986).

10. P. G. Hall, G. R. Davies, J. E. McIntyre, I. M. Ward, D. J. Bannister, and K. M. F. le Brocas, Polym. Commun., 27, 98 (1986).

11. R. Spindler and D. F. Shriver, J. Am. Chem. Soc., 220, 3036
(1988).

12. E. Tsuchida, N. Kobayashi, and H. Ohno, Macromolecules, 21, 3036 (1988).

13. G.-B. Zhou, I. M. Khan, and J. Smid, Polym. Commun., 30, 52 (1989).

14. K. Motogami, M. Kono, S. Mori, M. Watanabe, and N. Ogata, Electrochim. Acta, 37, 1725 (1992). 